\title{
Statistical Modeling of Antenna: Urban Equipment Interactions for LTE Access Points
}

\author{
Xin Zeng and Alain Sibille \\ COMELEC Department, TELECOM ParisTech, 75013 Paris, France \\ Correspondence should be addressed to Xin Zeng, xin.zeng@telecom-paristech.fr \\ Received 6 July 2012; Accepted 7 October 2012 \\ Academic Editor: Guangyi Liu
}

Copyright ( $\odot 2012$ X. Zeng and A. Sibille. This is an open access article distributed under the Creative Commons Attribution License, which permits unrestricted use, distribution, and reproduction in any medium, provided the original work is properly cited.

\begin{abstract}
The latest standards for wireless networks such as LTE are essentially based on small cells in order to achieve a large network capacity. This applies for antennas to be deployed at street level or even within buildings. However, antennas are commonly designed, simulated, and measured in ideal conditions, which is not the real situation for most applications where antennas are often deployed in proximity to objects acting as disturbers. In this paper, three conventional wireless access point scenarios (antenna-wall, antenna-shelter, and antenna lamppost) are investigated for directional or omnidirectional antennas. The paper first addresses the definition of three performance indicators for such scenarios and secondly uses such parameters towards the statistical analysis of the interactions between the wall and the antennas.
\end{abstract}

\section{Introduction}

With the development of new wireless communication systems and standards, antennas of base stations or access points are more likely deployed at street level in proximity to walls and urban furniture other than the traditional antennas for GSM networks which are mounted on rooftops with few neighboring disturbers [1-3]. The effect of surrounding disturbers may be strong or weak and is very sensitive to the antenna location and antenna characteristics. Unfortunately, antennas are commonly designed, simulated, and measured in isolation from anything else as much as possible, which is not a realistic use case. For instance, the radiation pattern of a dipole mounted beside a wall is highly perturbed, indicating the severe performance degradation to omnidirectionality (see below). Unfortunately, such disturbances are highly variable and impact the behavior of antennas in an uneasily predictable manner, with significant consequences in terms of cell coverage. The latter can be quantitatively evaluated through, for example, ray tracing of even empirical propagation simulation tools, but it will be very costly to compute the propagation for many realizations of disturbed antenna characteristics. For this reason, a reasonable approach is first to develop a statistical model for the antenna radiation behavior, before combining it with a model of simulation of the propagation. It is the goal of this paper, keeping in mind that statistical models lend themselves to the tuning of the trade-off between complexity and accuracy, depending on the number of parameters involved in the statistical distributions. For instance, [4] gives an example of this approach through radiation patterns series expansions. Actually, the evaluation of the impact of close objects on radiation is not entirely new. In [5-7], for example, the authors simulated and analyzed the antenna radiation patterns on roofs, beside walls, and on top of cars in a deterministic way. In [8-10] and many other papers, a work has been done in order to statistically evaluate the effect of, for example, a human body on antennas performance. Unfortunately and in spite of its relevance for small cells in wireless networks, little has been done regarding the interaction of antennas with walls or with urban furniture.

In the present paper, we investigate the effect of such influences on antenna radiation patterns, by using a statistical approach for their analysis. For this purpose, we first define the concept of ideal antenna sector, which can, for example, be related with the coverage area targeted by an antenna in a street. Three kinds of parameters relevant for quantifying the influence are proposed. Secondly, 


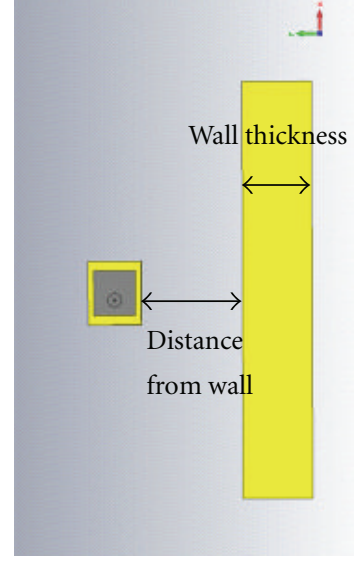

(a) Patch-wall perpendicular

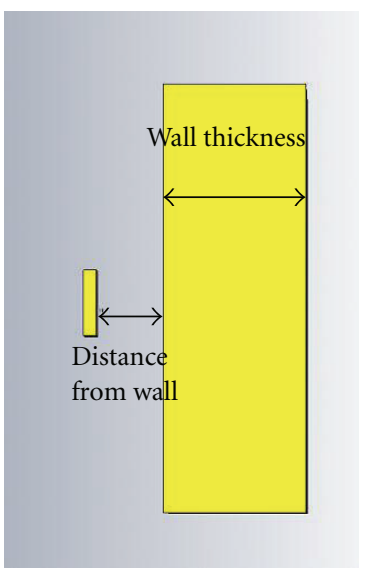

(b) Patch-wall parallel
FIGURE 1: Patch antenna locations and parameters.

three antenna-disturber interaction scenarios (antenna-wall, antenna-shelter of bus stop, and antenna lamppost) are investigated. The analysis in particular seeks to check whether a normal or lognormal distribution can provide an acceptable fit to the observed distributions, for example, for power gains. All results are obtained from detailed electromagnetic simulations of the environed antennas, using both CST [11] and WIPL [12] commercial tools.

\section{Sector Definition and Performance Parameters}

In relation with the placement and performance of an access point antenna, the designer mostly aims at ensuring that the main beam will cover the targeted directions, conditioned by the power gain to exceed a certain level $[13,14]$. This also assumes that the orientation and tilt angle will be steered adequately from just knowing the geometry of the antenna. In the present paper, we address both omnidirectional and directional antennas and we attempt to evaluate the impact brought by environmental perturbations to the radiated power, taking into account the considered "sector" (defined by azimuth and elevation widths). Ideally, a radiation sector is thus defined versus an intended use, that is, as a specification of the antenna. However, in practice, real antennas already cannot perfectly obey the specification. The deviation with respect to ideality occurs both on the beam widths and on the radiated power profile, with respect to the ideal profile (which is flat within the sector and null outside). A first aspect of antenna nonideality consequently would be to define performance criteria of an isolated antenna. However, this is not the purpose of the present work, whose goal is to address degradations by comparing isolated antennas. Also, from the perspective of an antenna designer, the main beam of an antenna shall include all the interested directions that perfectly match the sector requirements for an operator. For those reasons, the sector is going to be defined from the characteristics of the isolated antenna as the rectangle in angular coordinates given by the quarter power beam width of the isolated antenna ( $-6 \mathrm{~dB}$ from the maximum), in both
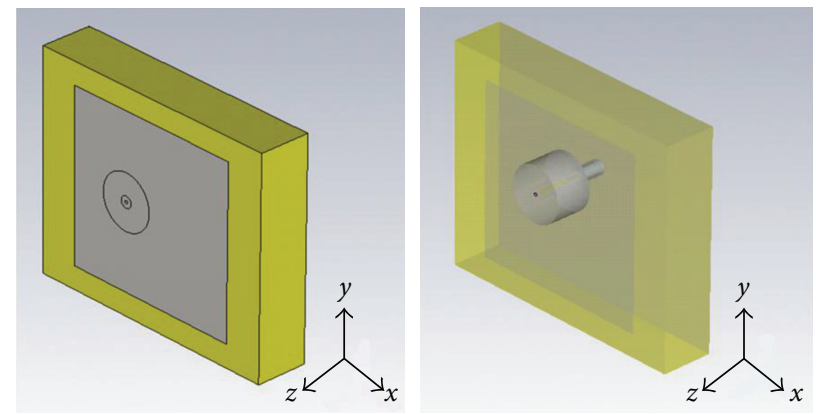

Figure 2: Patch antenna geometry.

E plane and $\mathrm{H}$ planes, completely defined by the elevation and azimuth angles $\theta_{\min }, \theta_{\max }, \phi_{\min }$, and $\phi_{\max }$. In the case of an omnidirectional antenna, only the elevation beam width needs to be defined and the parameters below are adapted accordingly.

In practice, this definition is still not fully sufficient, since the radiation pattern is frequency-dependent. Therefore, in the analysis conducted in Sections 3 and 4, a unique frequency independent antenna sector is chosen as the sector with the narrowest beam widths over the set of considered frequencies.

Given this definition of a sector, three important statistical parameters are proposed below, in order to quantify the capability of an antenna to radiate optimally into the considered sector. Beforehand, some quantities need to be defined.

$\mathrm{SE}_{\text {ref }}$ stands for sector efficiency as expressed in (1), where $G_{\text {ref }}(\phi, \theta)$ is the power gain of the isolated (reference) antenna along $\phi, \theta$ azimuth and elevation angles, respectively as follows:

$$
\mathrm{SE}_{\mathrm{ref}}=\frac{\int_{\phi_{\min }}^{\phi_{\max }} \int_{\theta_{\min }}^{\theta_{\max }} G_{\mathrm{ref}}(\theta, \phi) d \Omega}{\int_{0}^{2 \pi} \int_{0}^{\pi} G_{\mathrm{ref}}(\theta, \phi) d \Omega}
$$

This quantity basically expresses that not all radiated power falls within the sector, even for the isolated antenna, owing to the fact from elementary principles that a part of the radiation necessarily leaks out of the sector.

In the expressions below, $G(\phi, \theta)$ is the power gain of the disturbed antenna. $S_{\text {sector }}$ is the solid angle area of the sector.

The three performance parameters are as follows.

(i) The normalized "In Sector Power Gain" (ISPG) is

$$
G_{\text {norm }}(\theta, \phi)=\frac{G(\theta, \phi)}{\mathrm{SE}_{\text {ref }} \cdot \mathrm{TE}_{\mathrm{ref}}},
$$

where $\mathrm{TE}_{\mathrm{ref}}$ stands for total efficiency (matching efficiency + radiation efficiency) of the isolated antenna.

(ii) The normalized "In Sector Mean Power Gain" (ISMPG) within the sector is

$$
\mu_{\text {gain }}=\frac{\int_{\phi_{\min }}^{\phi_{\max }} \int_{\theta_{\min }}^{\theta_{\max }} G_{\text {norm }}(\theta, \phi) d \Omega}{S_{\text {sector }}} .
$$



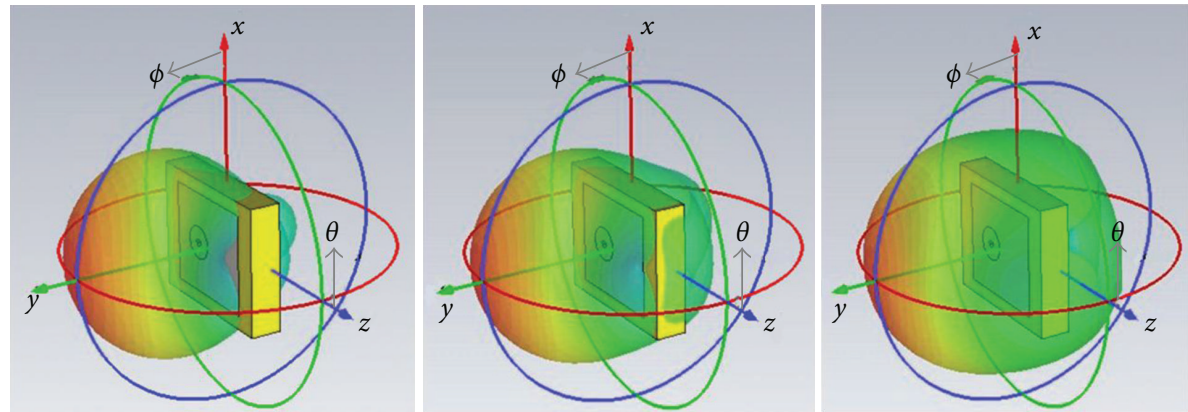

FIGURE 3: Patch antenna power radiation pattern at $1.71,1.905$ and $2.05 \mathrm{GHz}$.

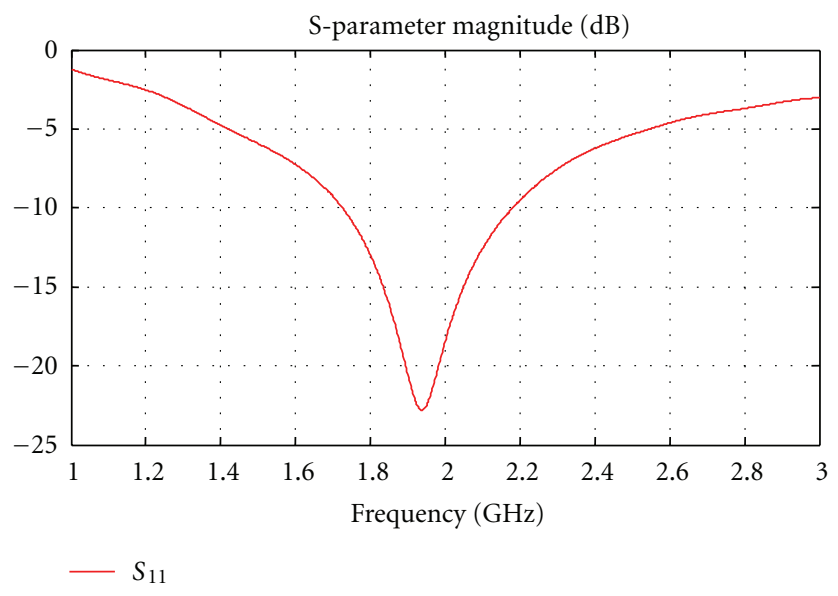

FIgURE 4: Patch antenna reflection coefficient.

$S E_{\text {ref }} \cdot T E_{\text {ref }}$ here is a normalization factor. Owing to this factor, $\mu_{\text {gain }}$ can thus be understood as the angular mean power gain over the sector, normalized by the isolated antenna sector efficiency and total efficiency. The reason for incorporating this normalization factor is that the sector efficiency and total efficiency turn out to depend on the frequency, even for the isolated antenna. By normalizing, we retain in $\mu_{\text {gain }}$ only the effects due to the disturbances rather than the non idealities of the isolated antenna.

(iii) The "In Sector Standard Deviation of the Power Gain" (ISSDPG) within the sector is

$$
\sigma_{\text {gain }}=\sqrt{\frac{\int_{\phi_{\min }}^{\phi_{\max }} \int_{\theta_{\min }}^{\theta_{\max }}\left(G_{\text {norm }}(\theta, \phi)-\mu_{\text {gain }}\right)^{2} d \Omega}{S_{\text {sector }}}} .
$$

Basically, these 3 parameters tell us if the radiated power is constant and sufficiently high within the sector, with respect to the isolated antenna. ISPG contains all values of the radiated power in all directions within the sector, thus it provides us with very detailed information about the antenna radiation characteristic. ISMPG tells us what is in average, the gain of the antenna within the sector, and whether it is higher than that of an isolated antenna. ISSDPG tells up to what extent the gain deviates from this mean value, in other words whether the radiation pattern is flat or varying within
TABLE 1: Parameters of patch-wall scenarios.

\begin{tabular}{lccc}
\hline Frequency $(\mathrm{GHz})$ & 1.71 & 1.905 & 2.05 \\
Permittivity of wall & 3 & 4 & 5 \\
Distance between patch (edge) and wall $(\mathrm{mm})$ & 50 & 100 & 150 \\
Wall thickness $(\mathrm{mm})$ & 100 & 150 & 200 \\
\hline
\end{tabular}

the sector. The cumulative distribution function (CDF) of these three parameters provides us with an indication on the gross characteristics of the variability of radiation patterns and they will be shown in the following sections.

\section{Antenna in Proximity to a Wall}

In this section, we consider the practically important case of an antenna placed on a wall.

3.1. Analysis of Directional Antenna-Wall Interaction. In the first part of this section, two patch-wall configurations are considered (Figure 1).

In order to simulate a real scenario, we designed an air patch antenna operating from $1.7 \mathrm{GHz}$ to $2.1 \mathrm{GHz}$ in proximity to a wall, whose characteristics are shown in Figures 2-4. Given that the power radiation pattern is getting narrower at low frequency, the sector at $1.7 \mathrm{GHz}$ is selected to cover the angles $\phi=[30-150], \theta=[55-150]$ in degrees according to the definition in Section 2. This patch antenna is a directional antenna, which may be deployed in two configurations: one is parallel to a wall in order to cover the area along a street; the other is perpendicular to a wall in order to cover a wide area, a street crossing, a square, and so forth.

The patch location and the wall parameters are shown in Figure 1 and in Table 1, respectively. The frequency points of the simulation are selected from $1.7 \mathrm{GHz}$ to $2.1 \mathrm{GHz}$ as one of the potential LTE bands. The permittivity of the wall has been chosen to vary from 3 to 5 , according to common building material parameters [15]. The other parameters are determined based on typical building structure and the experience of antenna deployment provided by an antenna commercial company. There are 81 realizations for each configuration and all electromagnetic simulations have all 


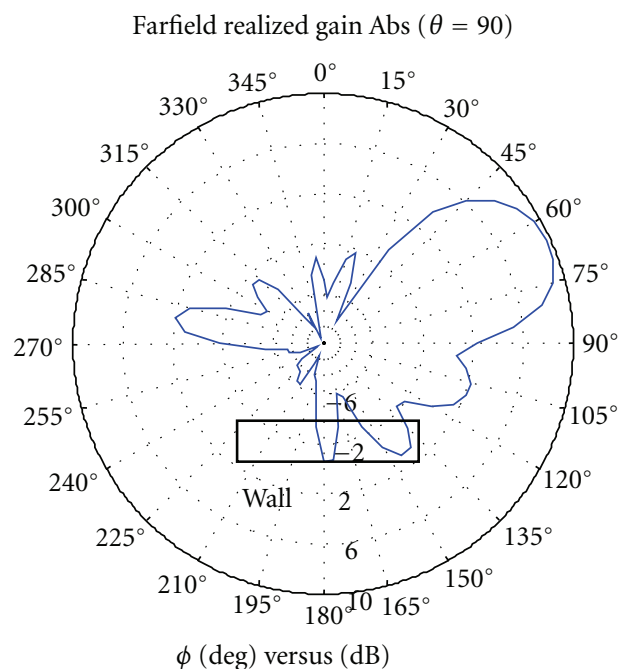

(a)

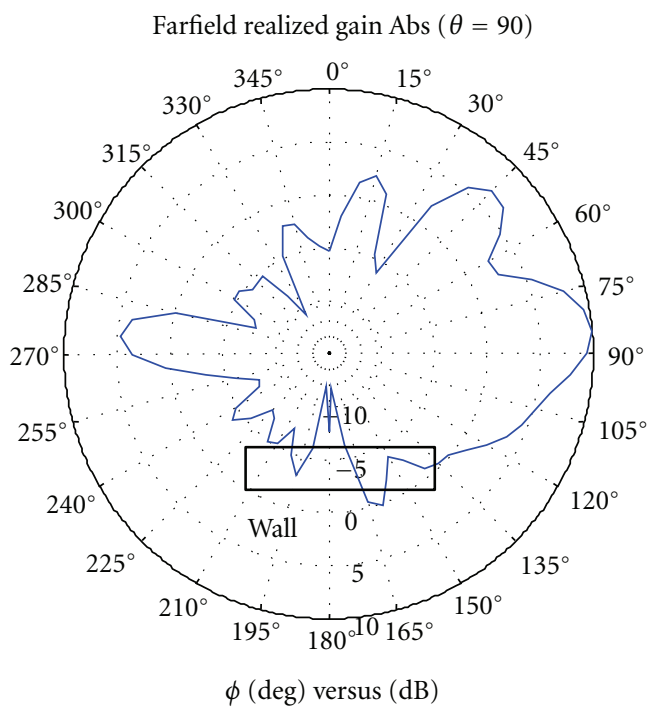

(c)

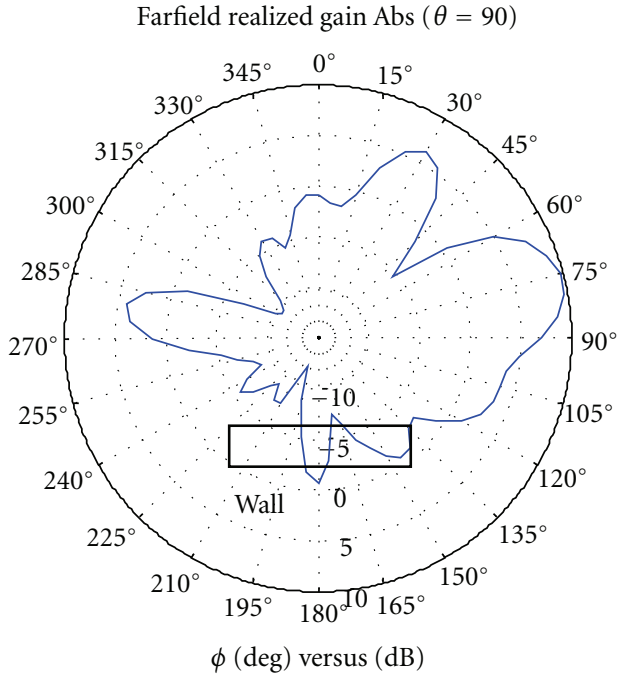

(b)

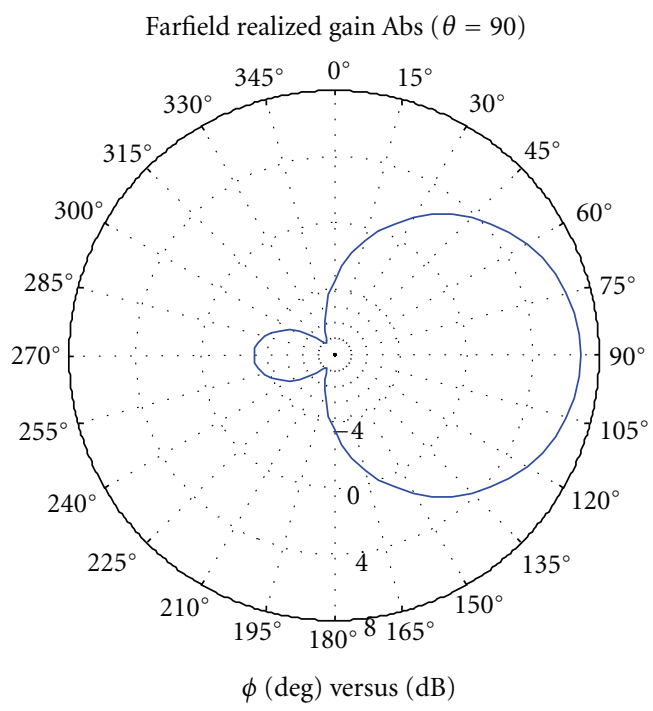

(d)

FIGURE 5: Power gain in azimuth in the perpendicular case (a)-(c) Wall distance $50 \mathrm{~mm}, 100 \mathrm{~mm}$, and $150 \mathrm{~mm}$; (d) isolated antenna $(1.7 \mathrm{GHz})$.

been carried out using CST. In the simulations, neither the antenna nor the materials are lossy. Thus, the considered effects are solely related to the impedance mismatch or (mainly) to the degradation of the radiation pattern shape.

Figures 5 and 6 reveal the serious impact of the wall on the patch antenna radiation patterns in both cases, even in the parallel case, which is not so intuitive since commonly it is assumed that the back radiation has little influence on the main beam. It can be found in Figure 5 that the main beam direction is driven away from the wall when the antennawall distance decreases. This phenomenon is obvious because the lower half of the original main lobe experiences a wall reflection and sums up with the other half. The smaller the distance, the more power the reflected by the wall and the larger the reflection angles. In order to represent the main beam direction and to know how it is affected by this effect, we use the mean radiation direction (MRD) proposed by Fleury [16], which is more stable than maximum gain direction. The MRD is based on the following vector:

$$
\mu_{\Omega}=\oint e(\phi, \theta) G(\phi, \theta) d \Omega,
$$

where $e(\phi, \theta)=[\cos (\phi) \sin (\theta), \sin (\phi) \sin (\theta), \cos (\theta)]^{T}$. From this mean vector, we can extract the MRD as the direction of $\mu_{\Omega}$.

Figure 7 highlights the distribution of the mean azimuth angle $\phi_{\text {mean }}$ over the set of realizations. Notice that the difference of MRD between the disturbed antenna and the isolated antenna is up to 32 degrees and varies inversely with the distance from wall. As opposed to the perpendicular case, the parallel case is almost symmetrical, thus the mean direction does not change significantly as compared to the isolated antenna, see Figure 6. 


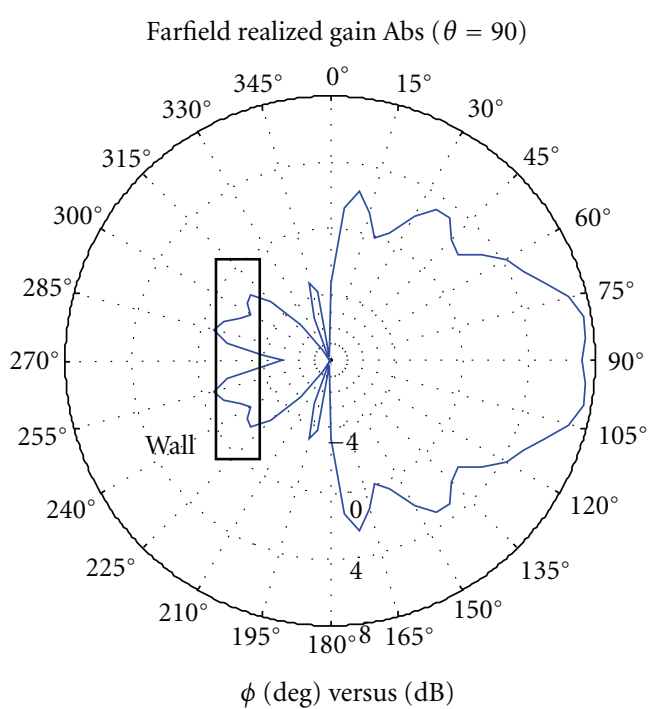

(a)

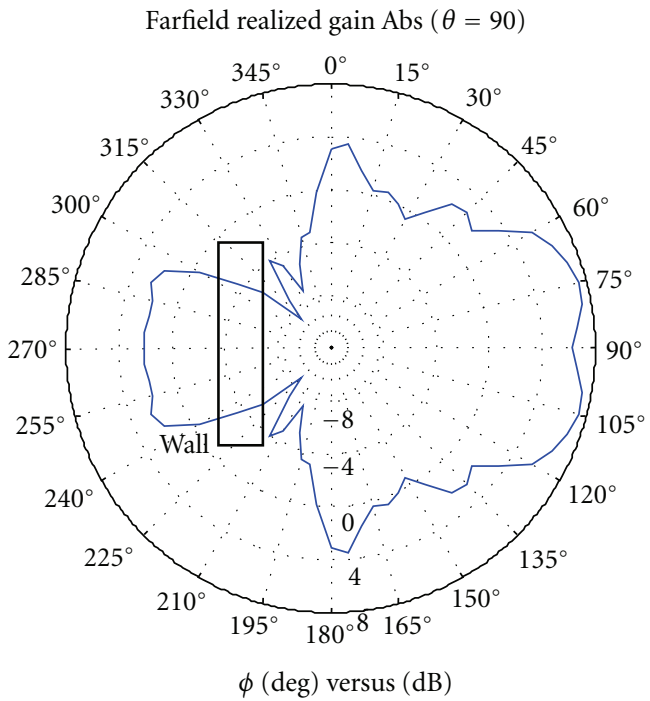

(c)

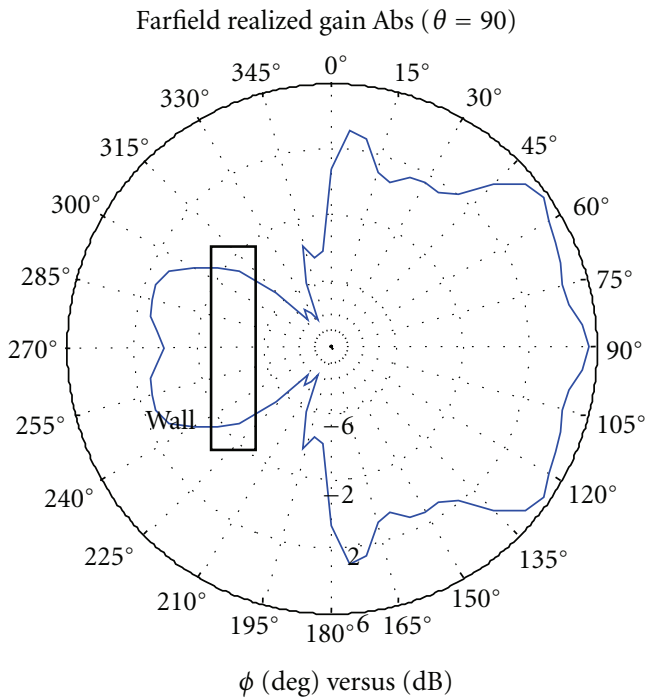

(b)

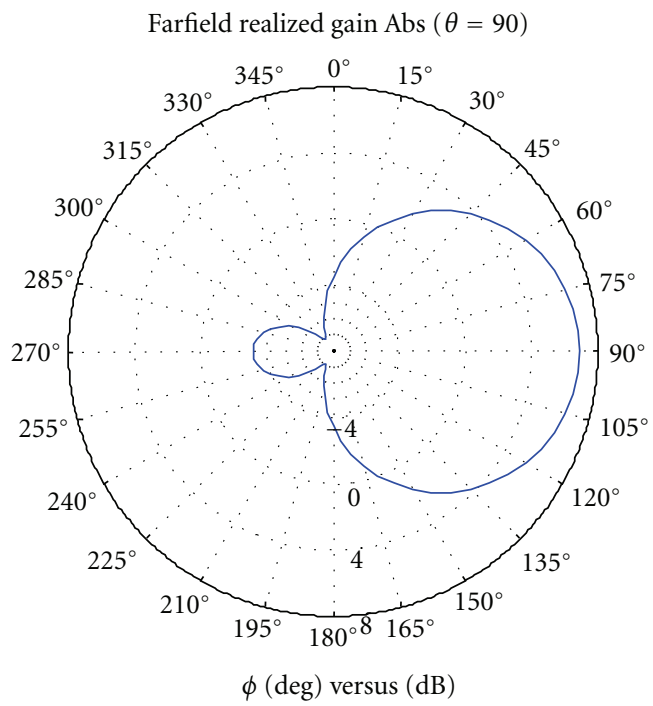

(d)

FIgURe 6: Power gain in azimuth in the parallel case. (a)-(c): wall distance $50 \mathrm{~mm}, 100 \mathrm{~mm}$, and $150 \mathrm{~mm}$; (d) isolated antenna (1.9 GHz).

Figures 8 and 9 show the aggregated ISPG distribution for the two cases, at each frequency and with all frequencies altogether. Owing to the total and sector efficiencies normalization, the main frequency dependence is removed and the statistics are better combined. The Gaussian bestfit distribution is plotted for comparison. Notice that in Figure 6, several nulls can be seen, which is due partly to the blocking of the radiation into the directions penetrating the wall, and partly to severe destructive interference as the result of reflections at the first and second interface of the wall, leading to the long tail in Figure 8 of low gain values. This effect will be more clearly seen in the omnidirectional antenna case. In other examples of antennadisturber interactions, such tails have been analyzed in terms of general extreme value distribution or of a Gaussian mixture distribution $[9,10]$.

Figure 10 shows the ISMPG for the former scenario. As expected, because the main lobe of patch is parallel to the wall and due to strong wave reflections out of the sector, the average gain within the sector is less than the isolated antenna. Notice that the shorter the distance from wall, the larger the portion of power reflected from wall to outside of the sector, which leads to less average gain as shown below. However, in the latter case, it is difficult to find a regular distribution of the average gain (Figure 11). While wall reflection should increase power within the sector, destructive interference (e.g., at $1.71 \mathrm{GHz}$ ) sometimes actually attenuates the gain in the sector.

Figure 13 shows the cumulative distribution of ISSDPG for the perpendicular scenario and Figure 14 shows the same parameter for the parallel scenario. Results from the three frequencies were combined in these two figures in order to construct a global statistical distribution. The deviation of the gain around the mean ranges from $2.7 \mathrm{~dB}$ to $5.9 \mathrm{~dB}$ in Figure 12 and from $1.6 \mathrm{~dB}$ to $3 \mathrm{~dB}$ in Figure 13. We observe that the Gaussian distribution fits the curves well, with the 


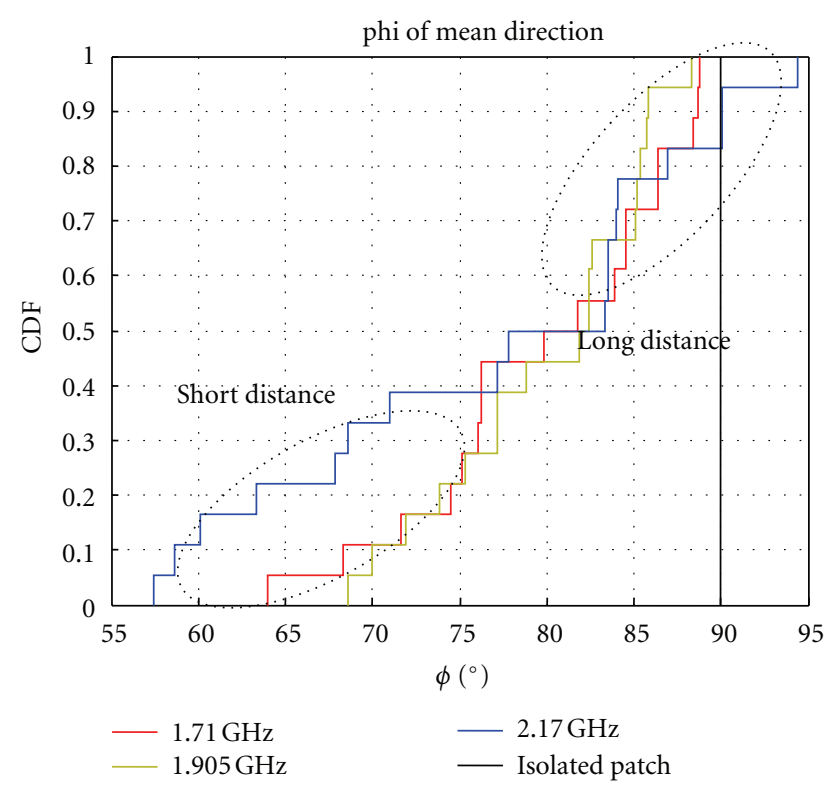

Figure 7: Distribution of the azimuth angle of the MRD for the patch-wall perpendicular case.

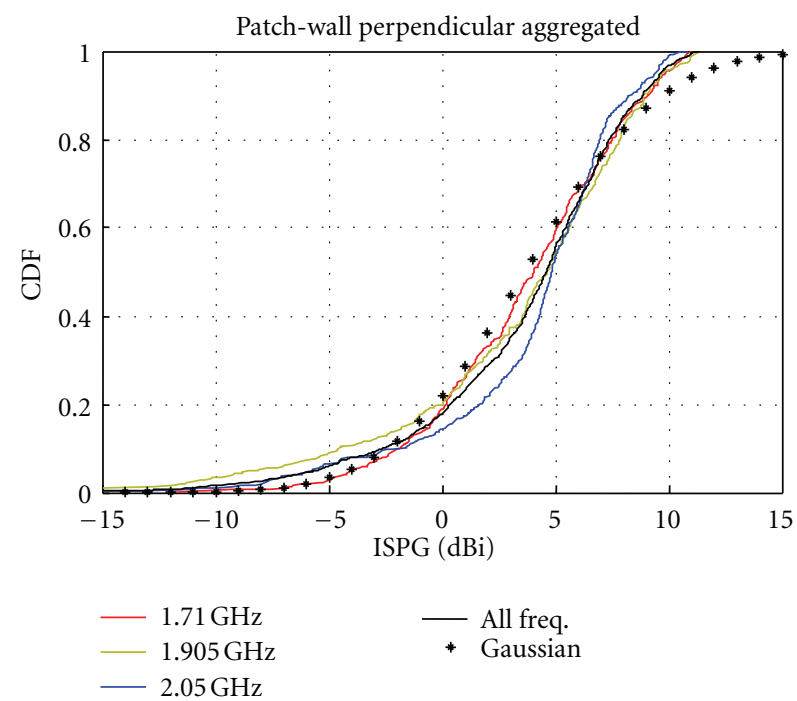

FIGURE 8: Distribution of ISPG for the perpendicular patch-wall case.

average and standard deviation $(\mu, \sigma)$ of $(4.12,0.84) \mathrm{dB}$ for the first scenario and $(2.26,0.29) \mathrm{dB}$ for the second scenario. The average of the Gaussian distribution in the first scenario is larger than that in the second one because when the patch is perpendicular to a wall, the wall reflects the radiated power from the lower half of the sector into the upper half, weakening the former and enhancing the latter. This leads to a large difference between the lower and upper halves of the pattern.

3.2. Analysis of Omnidirectional Antenna-Wall Interaction. For the second set of simulations, we considered a dipole vertical array, with a metallic supporting post attached to a wall as shown in Figure 14, again operating within the same

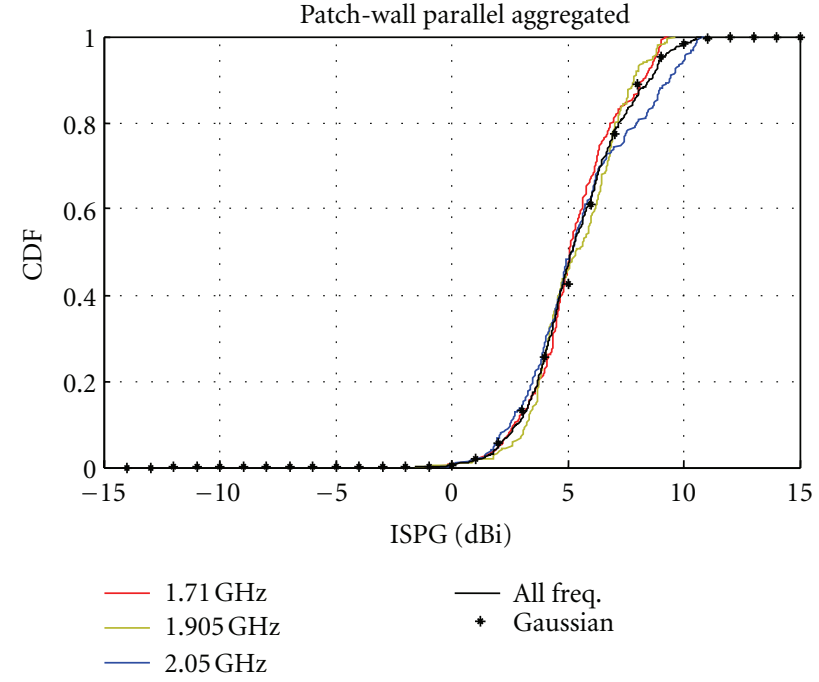

FIGURE 9: Distribution of ISPG for the parallel patch-wall case.

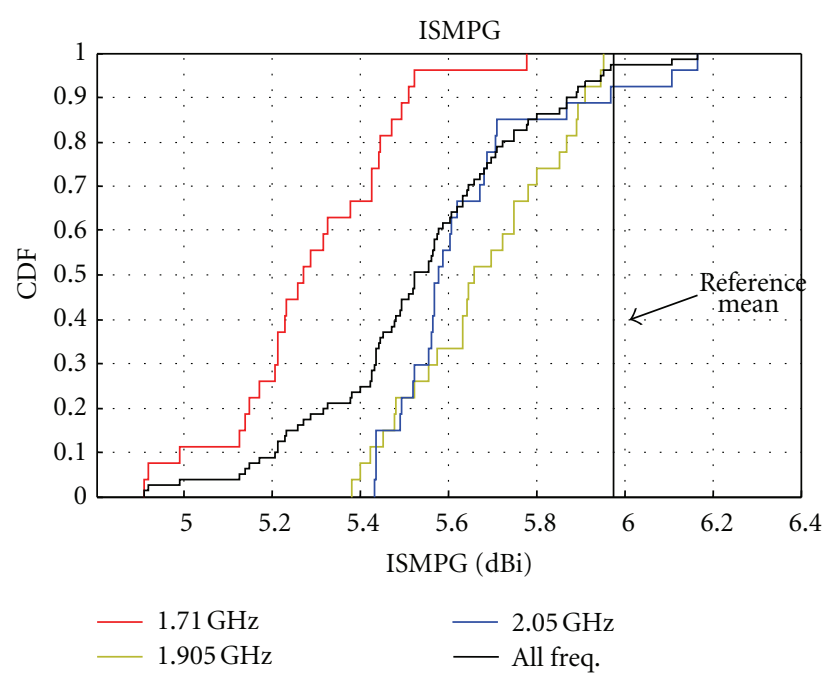

FIGURE 10: ISMPG distribution over all realizations for the patchwall perpendicular scenario.

TABLE 2: Parameters of dipole-wall scenarios.

\begin{tabular}{lccc}
\hline Frequency $(\mathrm{GHz})$ & 1.71 & 1.905 & 2.05 \\
Permittivity of wall & 3 & 4 & 5 \\
Distance between patch (edge) and wall $(\mathrm{mm})$ & 100 & 200 & 300 \\
Wall thickness $(\mathrm{mm})$ & 100 & 150 & 200 \\
\hline
\end{tabular}

bandwidth in order to compare with the directional antennas case. The simulation parameters are listed in Table 2, again providing 27 realizations at 3 frequencies. Since the radiation pattern of the dipole array is omnidirectional on the azimuth plane, the sector for the dipole array only restricts the elevation angle $(\theta=[65,115])$ according to its $-6 \mathrm{~dB}$ beam width.

Firstly, the azimuth pattern and distribution of ISPG are presented in Figures 15 and 16. The long tails of the 


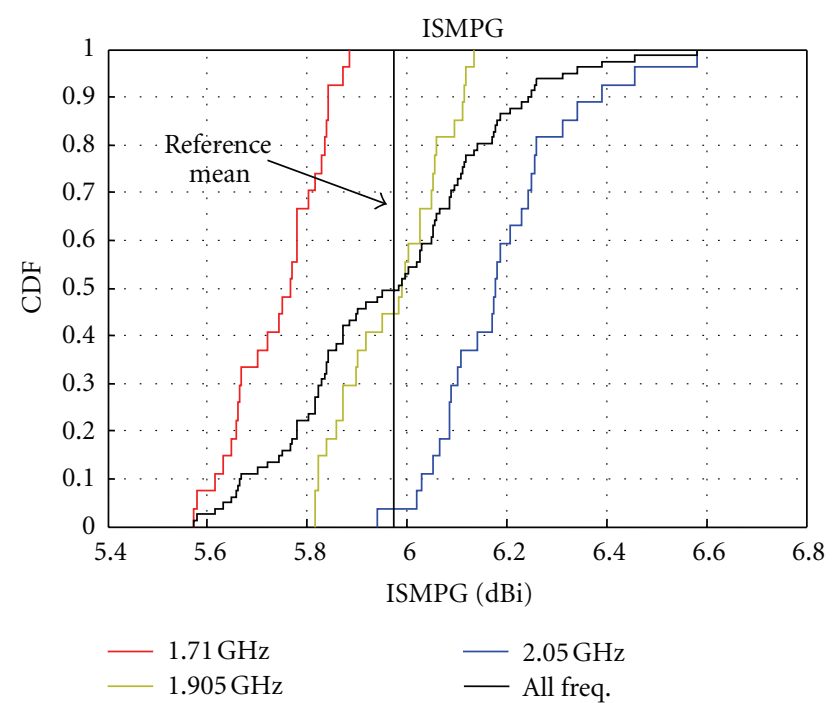

FIGURE 11: ISMPG distribution over all realizations for the patchwall parallel scenario.

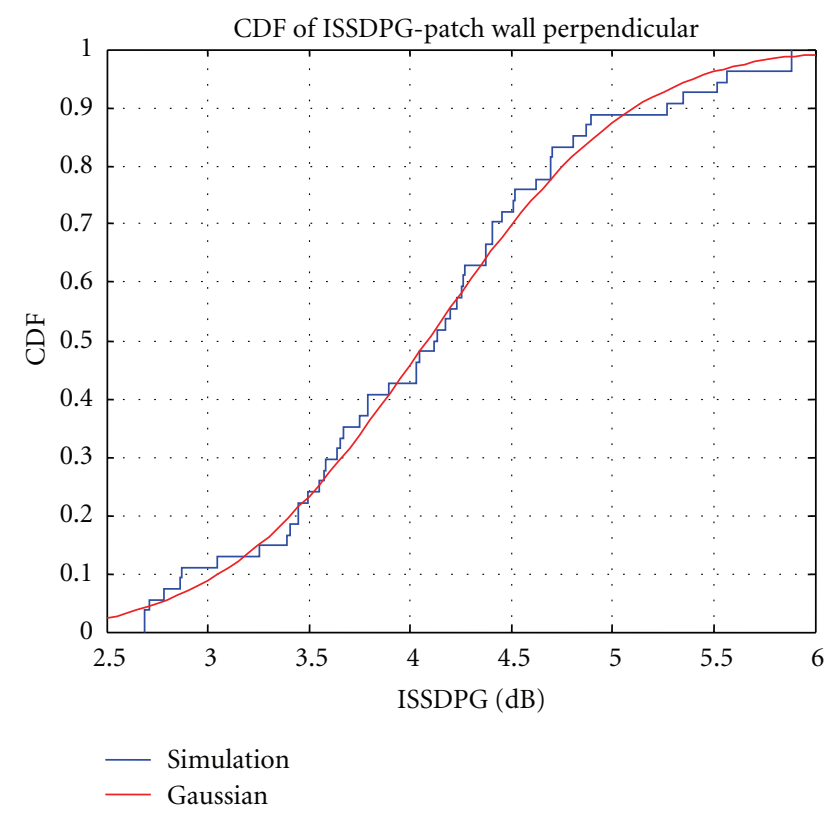

FIGURE 12: ISSDPG distribution for the patch-wall perpendicular case.

CDF, which are similar to the patch-wall scenario, are due to several radiation pattern nulls, as revealed in Figure 15. Some of the waves radiated towards the wall reflect on its near surface while some penetrate the wall and reflect on the second interface. These two reflections, together with the direct waves, cause constructive and destructive interference and form irregular patterns. Secondly, even though the wall disturbs the radiation patterns dramatically, most of the power radiates around the azimuth plane, not only resulting in unchanged average gain in the sector (not shown here) but also resulting in a large deviation around the mean. Figure 17 shows the CDF of ISSDPG at all frequencies, revealing that a normal distribution again fits the data quite well. The

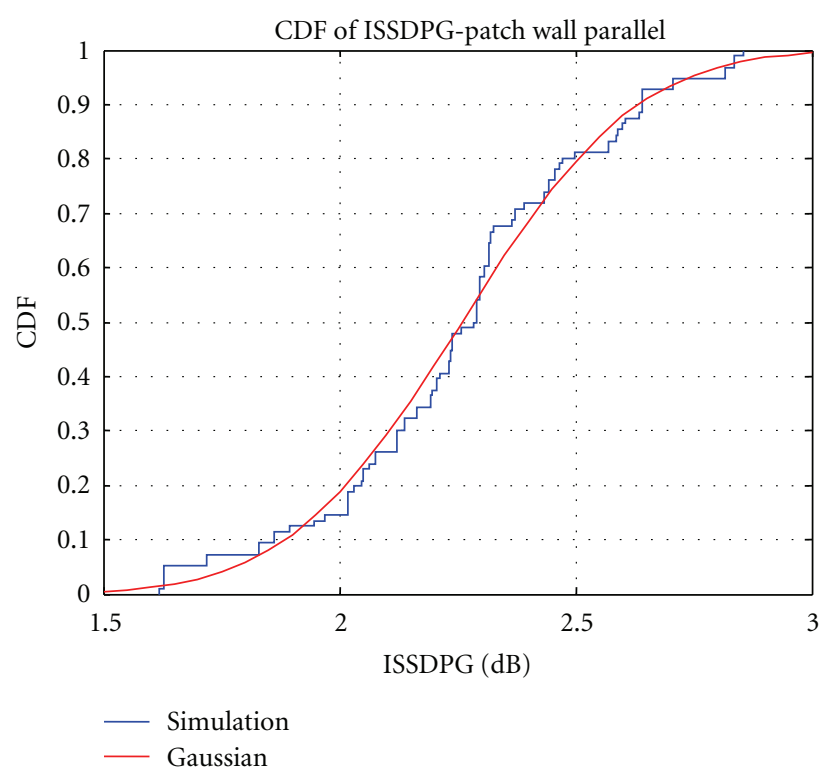

FIGURE 13: ISSDPG distribution for the patch-wall parallel case.

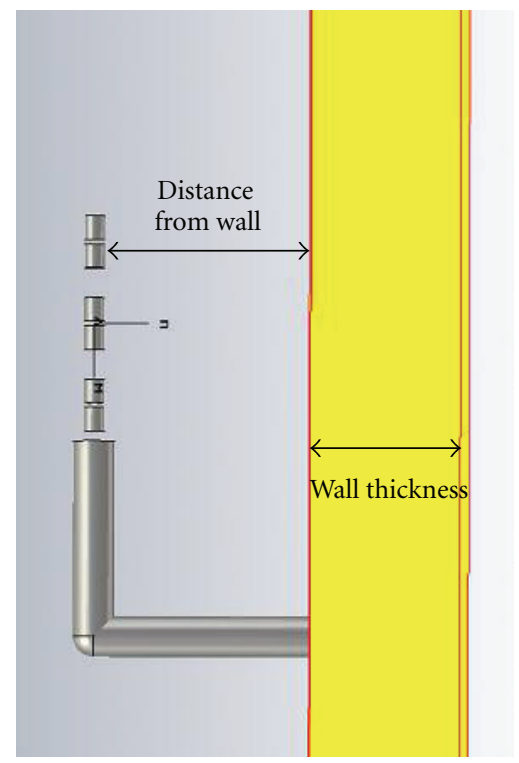

FIgURE 14: Dipole array locations and parameters.

parameters of the fitted Gaussian distribution are $(\mu, \sigma)=$ $(4.08,0.29) \mathrm{dB}$, which tells us that the standard deviation of gain in each pattern is not heavily dependent on the particular realization. A large ISSDPG means that the gain values within the considered sector are deeply disturbed and do not behave so nicely omnidirectionally as for the isolated antenna, therefore the antenna performance is degraded.

\section{Antenna Deployment on Urban Furniture}

Among the deployment schemes considered for $4 \mathrm{G}$ wireless networks, small cells and femtocells as well as relays are the most widely advocated. This implies bringing antennas very close to the users and as much invisible as possible. Then the 


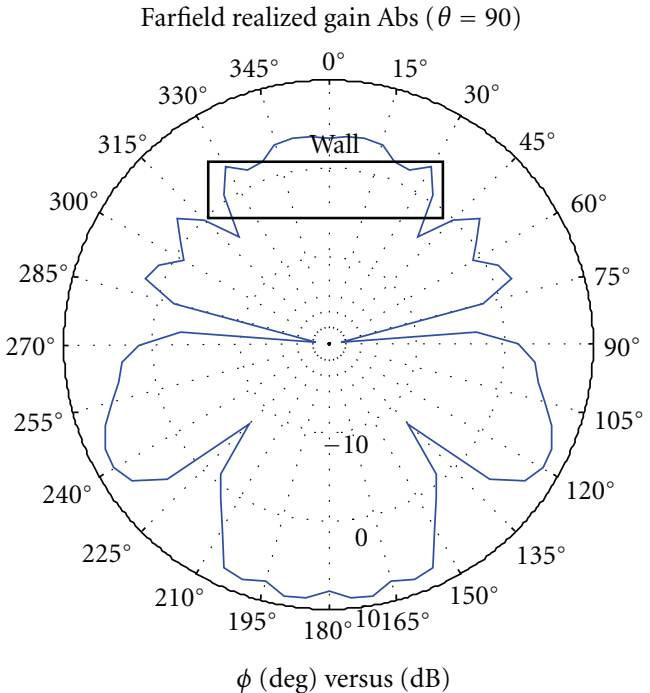

(a)

Farfield realized gain Abs $(\theta=90)$

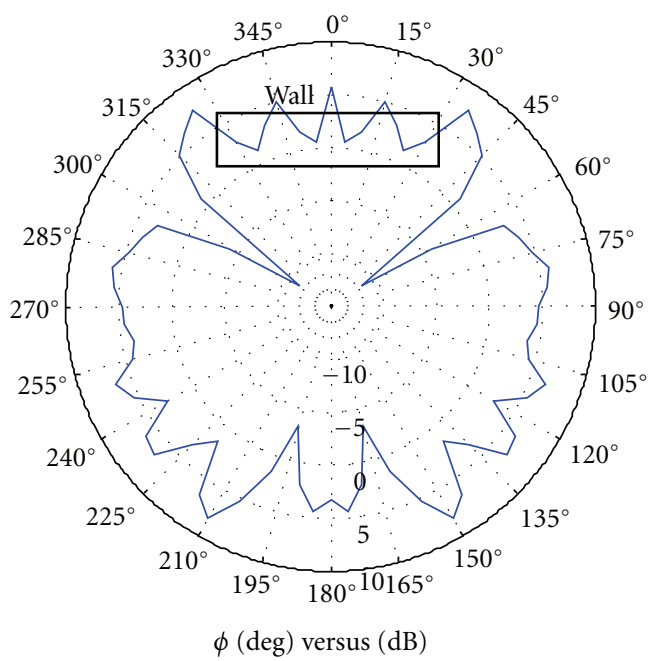

(c)

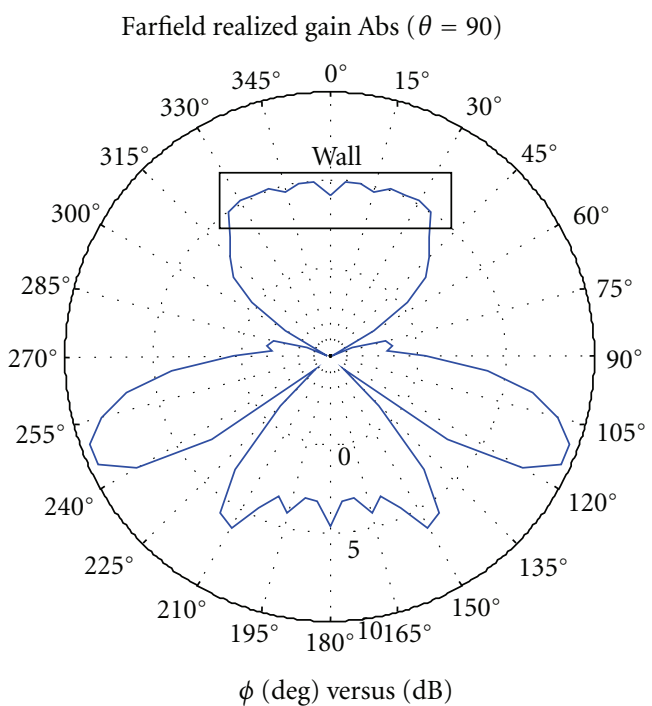

(b)

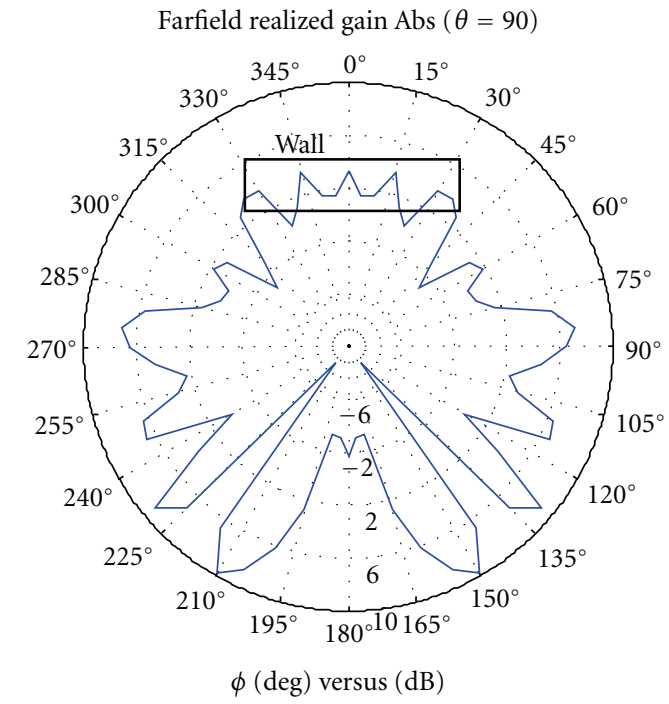

(d)

FIGURE 15: Examples of power gains in the azimuth plane for four realizations.

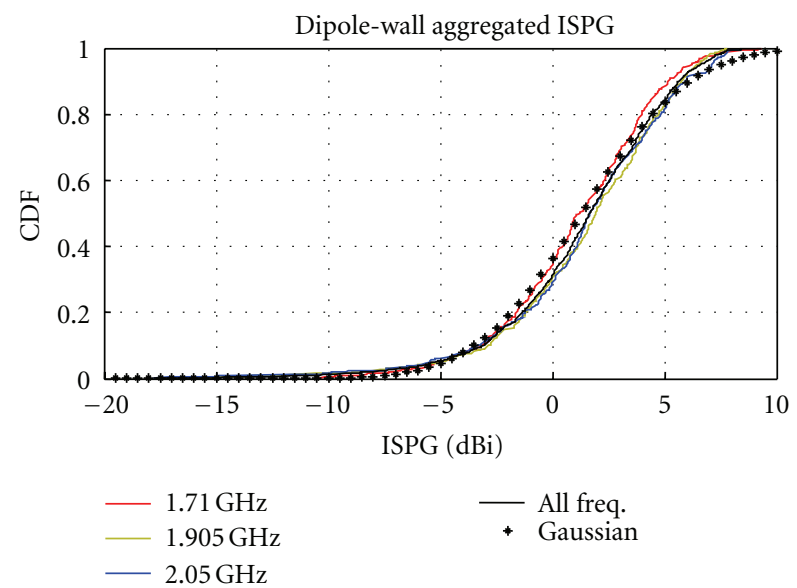

FIgURE 16: ISPG distribution for these two examples. 


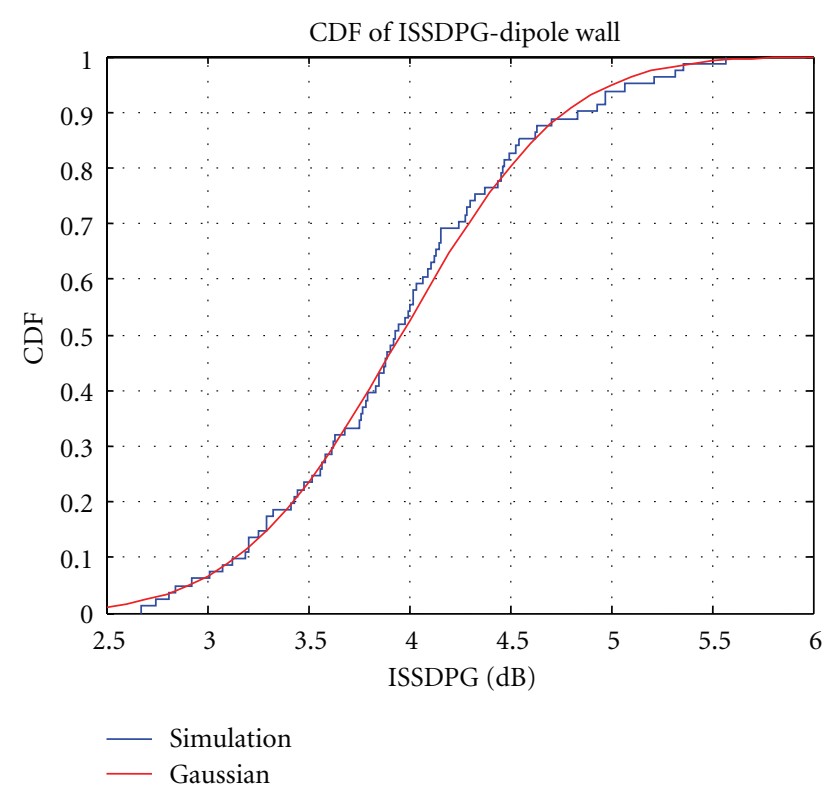

FIGURE 17: ISSDPG distribution for the dipole-wall scenario.

integration of the antennas into urban furniture is a serious option. Indeed, such equipments are very common in all modern cities, being part of transportation or other public services and it will likely be possible to contract with servicing companies for installing and maintaining $4 \mathrm{G}$ networks antennas. In addition, these pieces of equipment are often connected; however, as indicated above, urban furniture may cause a strong degradation of antenna performance, resulting in uncontrolled changes of the radiation patterns. In order to investigate this quantitatively, two scenarios are considered here, one being an omnidirectional antenna settled on a bus-stop shelter with a dielectric roof, see Figure 18, and a directional antenna attached to a lamppost (Figure 23).

These designs have been inspired by a real bus shelter and real lamp posts.

\subsection{Analysis of Omnidirectional Antenna-Shelter Interaction.} In this part, the simulated antenna is an ultra-wideband (UWB) bicone, designed to operate from $200 \mathrm{MHz}$ to $3 \mathrm{GHz}$ in Figure 19.

As opposed to the above cases, where the considered frequencies only ranged from $\sim 1.7$ to $2.1 \mathrm{GHz}$, this scenario considers a much broader band from 200 to $3000 \mathrm{MHz}$. The reason stems from the large variation of frequency bands contemplated for LTE, in some cases going down to $400 \mathrm{MHz}$ (e.g., for private mobile radio applications used by civil security services, police forces, etc.).

The other parameters are

(1) frequency points: 200 to $3000 \mathrm{MHz}$ every $200 \mathrm{MHz}$;

(2) permittivity of plastic roof: 3 ;

(3) size of roof $(\mathrm{mm}): 1800 * 810 * 3.6$.

The UWB antenna was assumed to be located on top of a bus-stop roof, according to a uniform spatial distribution. In practice, the antenna locations are distributed over

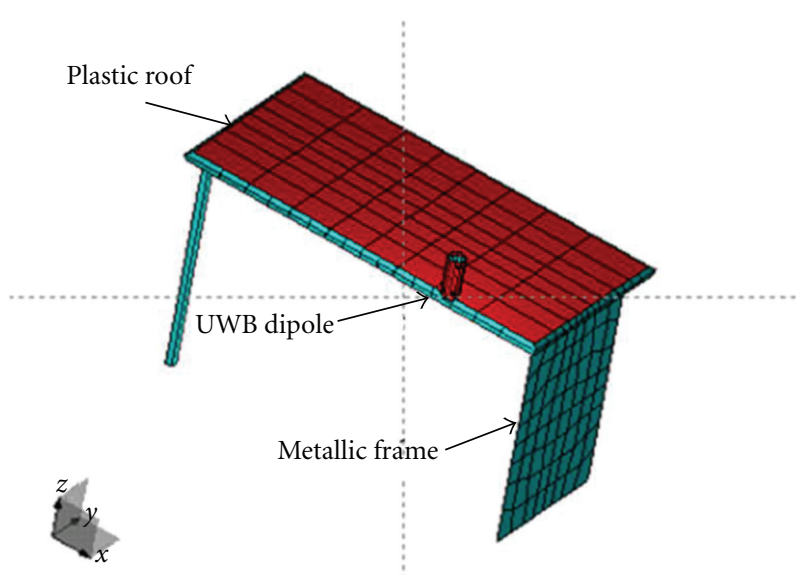

FIGURE 18: Antenna-urban equipment scenarios.

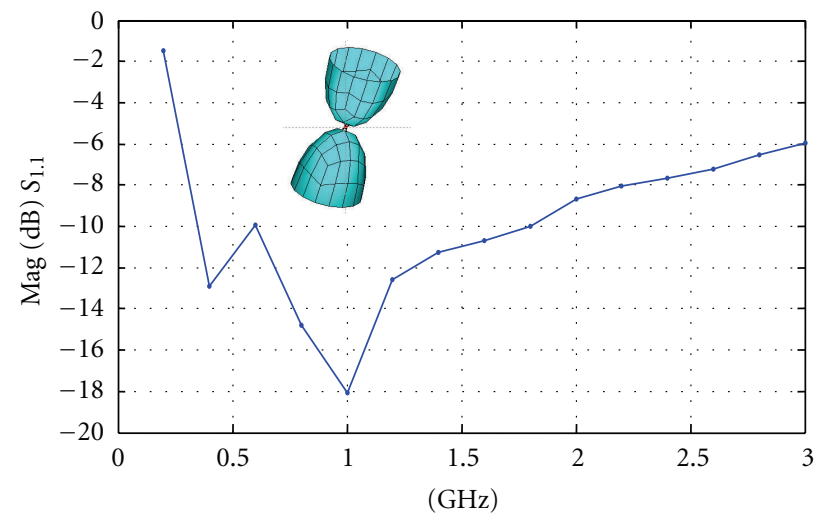

Figure 19: Design and reflection coefficient of an ultra-wideband omnidirectional radiator.

a rectangular grid, of increment $450 \mathrm{~mm}$ in length and $202.5 \mathrm{~mm}$ in width. This resulted in 25 antenna locations for each frequency point. All simulations were carried out using WIPL-D.

Owing to the UWB antenna's nearly perfect rotational symmetry, the isolated antenna pattern is extremely omnidirectional. It is also quite broad in elevation, although starting from $\sim 1.2 \mathrm{GHz}$ onward, it starts being multimode, which translates into a structured shape in elevation. This phenomenon is responsible for a slightly lower gain in the horizontal plane at these frequencies.

In the previous section, the statistical parameters at different frequencies have been aggregated together. However, in this section, the statistical parameters will be shown in individual frequency points in order to fully reveal a possible regular variation with frequency. Besides, since the "sector" means the solid angle area in which we are interested or where the antenna intends to radiate, the range of the sector has been adjusted in order to observe the variation of the ISPG. The sector is found to cover the angles $\phi=(0-360)$, and $\theta=(35-145)$ in degrees, so we will show results with sector $\phi=(0-360)$, and $\theta=(35( \pm 10) \sim 145( \pm 10))$.

The left column of Figure 20 exhibits the ISPG distributions for the antenna-shelter scenario at each frequency, 

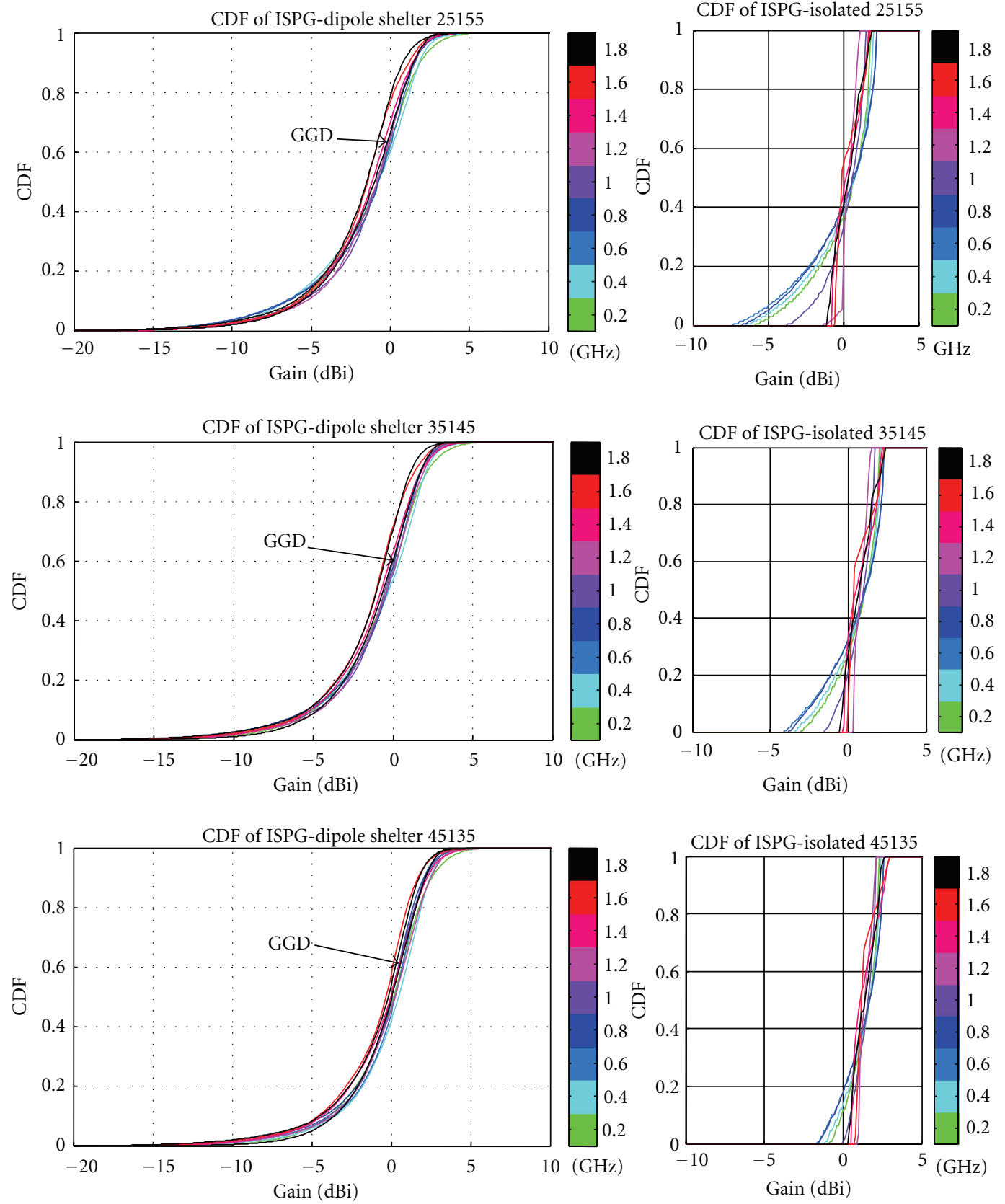

Figure 20: ISPG distribution for antenna-shelter and various sector widths in elevation: $\left(25-155^{\circ}\right),\left(35-145^{\circ}\right)$, and $\left(45-135^{\circ}\right)($ from top to bottom).

while the right column shows the corresponding ISPG distributions for the isolated antenna. Naturally in that case, the distribution is very narrow and only stems from the shape of the radiation pattern, which is not isotropic. In Figure 20, three values for the sector elevation beamwidth have been chosen $\left(130^{\circ}, 110^{\circ}\right.$, and $90^{\circ}$ from top to bottom) in order to test the robustness of the distributions to this parameter.

With the normalization of TE and SE, the curves at different frequencies are much closer and easier to compare. By looking at the isolated antenna ISPG plots, we see that the low gain tail is shorter when the sector gets narrower, especially for the low frequencies (green curves). This is because in a broader sector, more low power gain values originating from high angles in the radiation patterns are involved at low frequencies. A high frequency often means a broad beamwidth. Even though the first sector is the broadest, it still cannot catch many low power values of the gain. Thus, for high frequencies, the radiation patterns change less with the restriction of the sector.

The ISPG distributions for the disturbed antenna case, in contrast, vary less when the size of the sector decreases, which also proves the robustness of the selection of the sector. Since the distribution of ISPG is naturally asymmetrical due to the fact that the power gain can be extremely low 


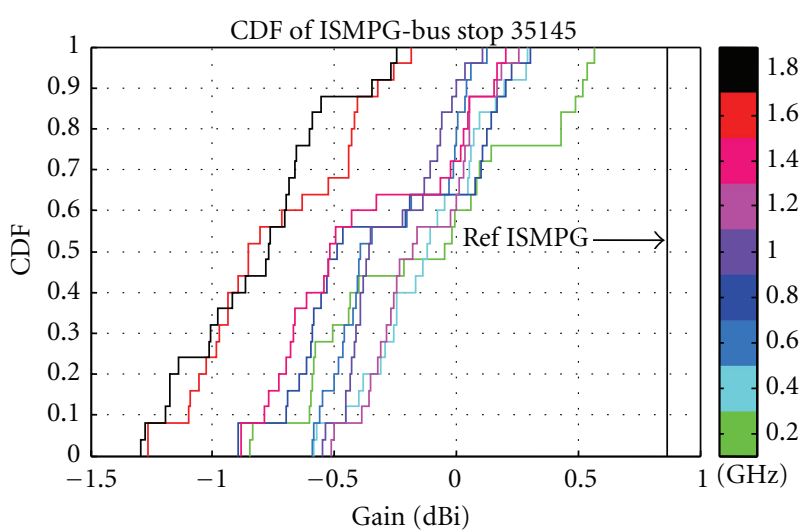

FIGURE 21: ISMPG distribution for antenna-shelter (standard sector).

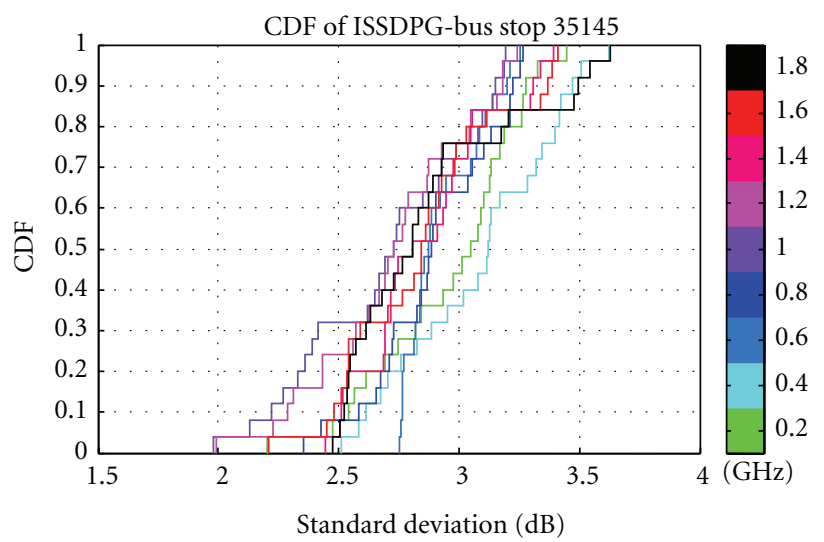

FIGURE 22: ISSDPG distribution for antenna-shelter (standard sector).

(nulls) but cannot be extremely high, Gaussian distribution is not suitable for this case, even though it performs well in previous scenarios. Generalized Gaussian distribution (GGD), whose shape parameter can be used to introduce skew, has been proposed to fit the ISPG distribution. After the normalization, the curves are found to follow the generalized Gaussian distribution far better than the Gaussian distribution. The goodness of fit will be studied in a future work.

Figure 21 reveals a negative impact by the dielectric roof, where the UWB dipole at all frequencies performs a little worse than the isolated antenna. With the increase of frequency, the ISMPG deviates from the reference with a difference up to $2.3 \mathrm{~dB}$. A high frequency generally translates into a more severe degradation than a low frequency. From the perspective of radiation main lobes orientation, the roof does not block radiations downward, thus signal detection for users below the roof is still achievable. The ISSDPG distributions are shown in Figure 22, revealing that the metallic frame largely increases the variance of the ISSDPG. The average value and difference of ISSDPG at one frequency is more than $2.5 \mathrm{~dB}$ and $1 \mathrm{~dB}$, respectively. The metallic frame creates constructive and destructive inference by its strong reflections, increasing ISSDPG and weakening the stability of radiation within the sector.
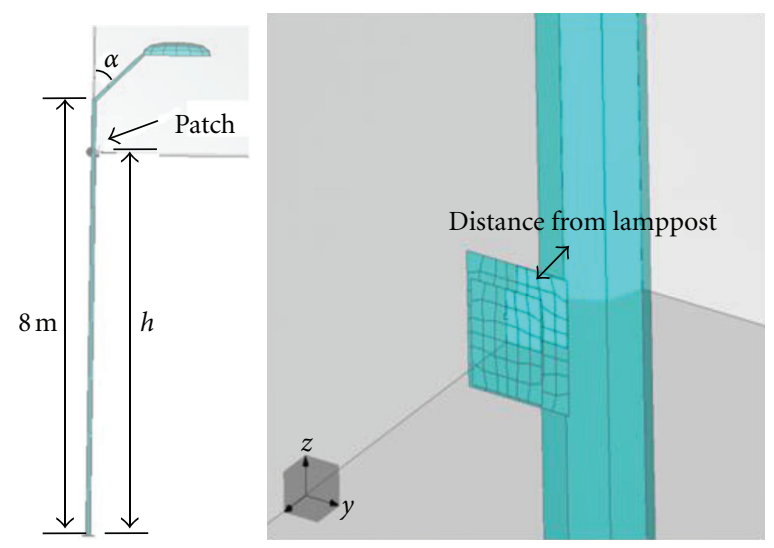

Figure 23: Antenna and lamppost location.

TABle 3: Parameters of dipole-wall scenarios.

\begin{tabular}{lccc}
\hline Frequency $(\mathrm{GHz})$ & 1.7 & 1.9 & 2.1 \\
Antenna height $(\mathrm{mm})$ & 7000 & 7400 & 7800 \\
Angle of corner $\left(^{\circ}\right)$ & 45 & 60 & 90 \\
Distance from lamppost $(\mathrm{mm})$ & 20 & 50 & \\
\hline
\end{tabular}

4.2. Analysis of Directional Antenna Lamppost Interaction. In order to simulate the scenario where a directional antenna is deployed on a lamp post, a simulation environment has been established as shown in Figure 23. Similar to the directional antenna-wall scenario, there are 3 kinds of input parameters (listed in Table 3), where the material of the lamp post is taken to be a PEC (perfect electric conductor). The variation of parameters represents the possible position and possible type of lamp post on which the LTE access point will be deployed.

As shown in Figure 24, because of the reflection of radiated waves by the metallic lamp post, the beam width becomes narrower whatever the frequency is. In contrast to the isolated antenna, the main beam of the disturbed antenna at $2.1 \mathrm{GHz}$ is almost allocated in the section, leading to a high ISPG after normalization, which implies high sector efficiency improvement at $2.1 \mathrm{GHz}$ under disturbance. This effect can be seen easily in the CDF of the ISPG (Figure 25).

For isolated antenna, the sector efficiency at $2.1 \mathrm{GHz}$ is 0.36, meaning a low normalization factor. But for disturbed antenna, the main beams at three frequency points are all focused in the sector. In other words, the sector efficiency has been highly improved especially at $2.1 \mathrm{GHz}$, than divided by a low normalization factor. Thus it interprets why ISPG at $2.1 \mathrm{GHz}$ is larger than the reference (isolated) antenna. Meanwhile, one can observe that the ISPG at $1.7 \mathrm{GHz}$ is smaller than the reference because the interaction with lamp post raises antenna's input return loss $\left(S_{11}=-3.69 \mathrm{~dB}\right)$. Such an unfavorable return loss impairs and weakens the radiated power in the sector, leading to low ISPG.

A fitted generalized Gaussian distribution curve has been plotted, for comparison with the aggregated ISPG CDF curve. However, the curves for individual frequencies are too far away from each other, only modeling the aggregated curve 

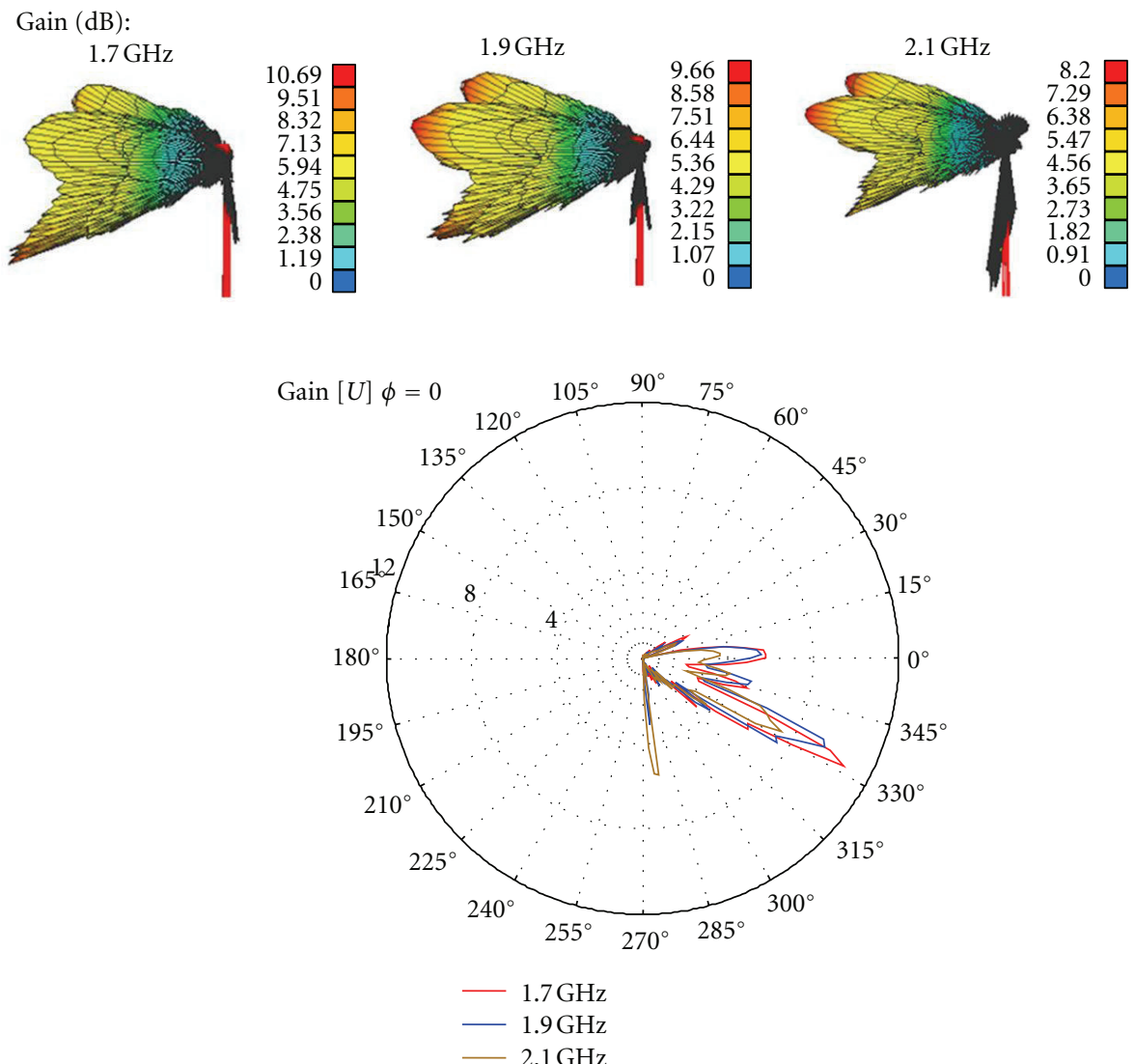

Gain $[U] \theta=-26$

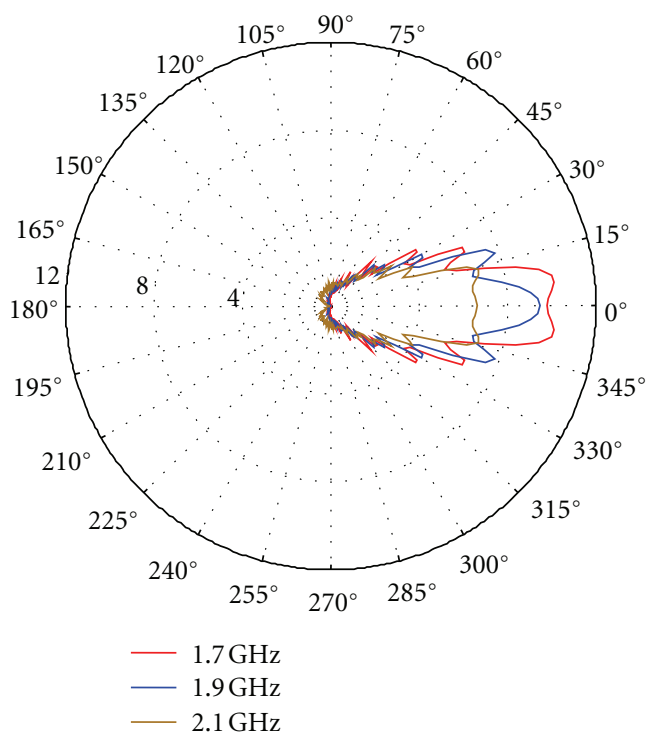

FIGURE 24: Radiation patterns for one realization in the lamppost scenario.

is not sufficient to find frequency-specific distribution while the ISMPG and ISSDPG at individual frequency are also very important to be modeled. The ISMPG (Figure 26) for three frequencies with very small variations is distributed around the reference ISMPG as expected. This means that the sector efficiency or the portion of power radiated within the desired direction at 1.9 and $2.1 \mathrm{GHz}$ is much better than the isolated antenna, and this quality does not change too much with the shape of lamp post or height of antenna due to a small variation.

With the lampshade on top of the antenna, the radiation upward will be reflected to ground, causing a strong beam downward and a null upward, which can be observed in Figure 24. Due to such a large variation of radiated power gains, the ISSDPG must be large, as shown in Figure 27. Again, because of the normalization factor and the low 


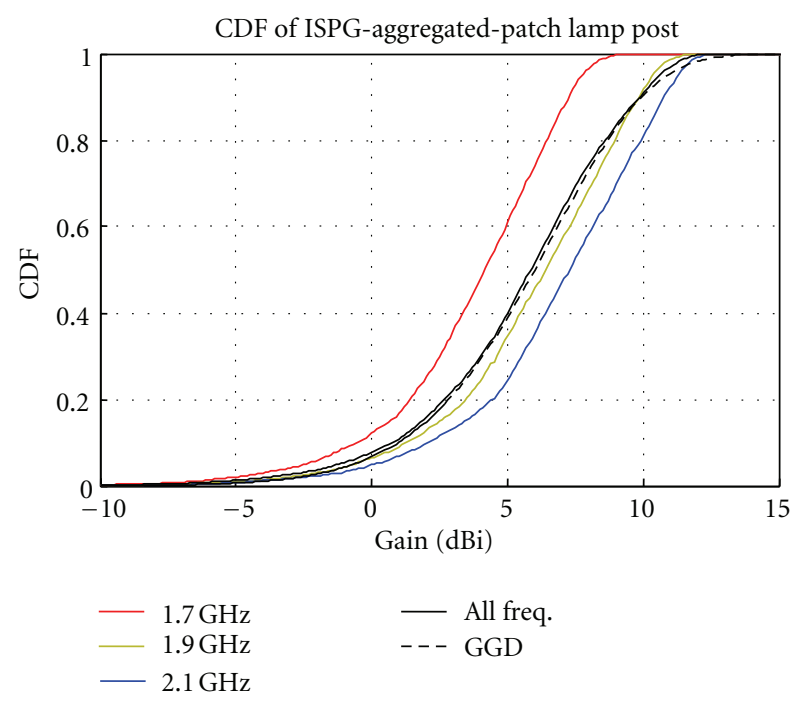

FIGURE 25: ISPG distribution for patch-lamppost scenario and fitted Gaussian distribution.

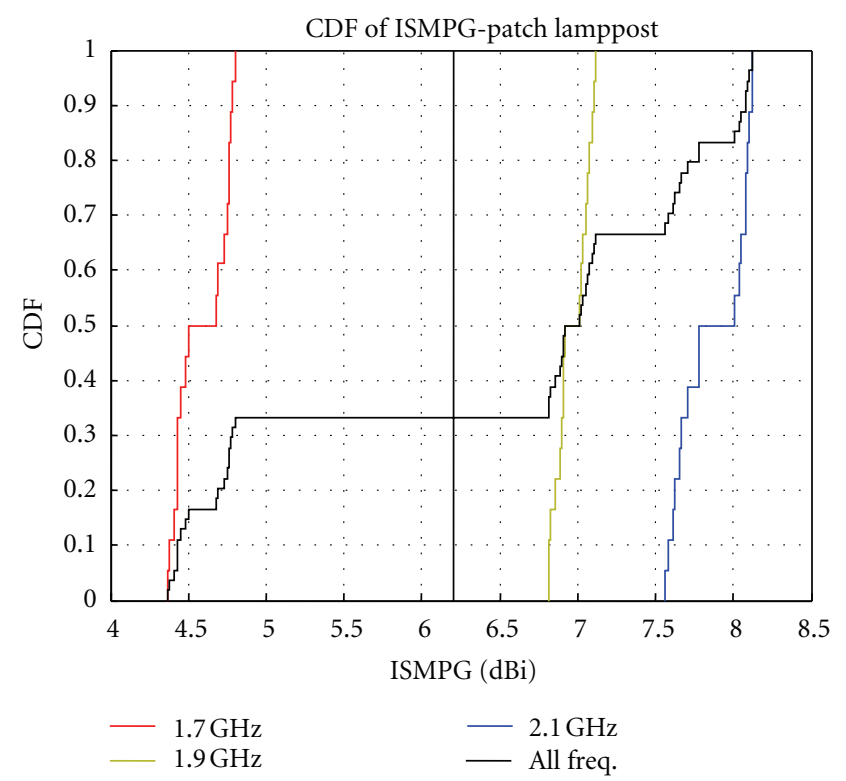

FIGURE 26: ISMPG distribution for patch-lamppost scenario.

$S_{11}$ at $1.7 \mathrm{GHz}$, the curves are separated. A fitted Gaussian distribution has been plotted to illustrate that the aggregated ISSDPG is hard to be modeled by Gaussian distribution. The first or second central moment of ISPG (ISMPG or ISSDPG) cannot be extremely low or high in this scenario, so their values have upper and lower boundaries which may be correctly described by a linear model (uniform distribution).

\section{Conclusion}

In this paper we have presented simulations' results on the performance of omnidirectional and directional antennas, in proximity to a wall, a bus shelter, and a lamp post, as examples of access point placement for $4 \mathrm{G}$ access points,

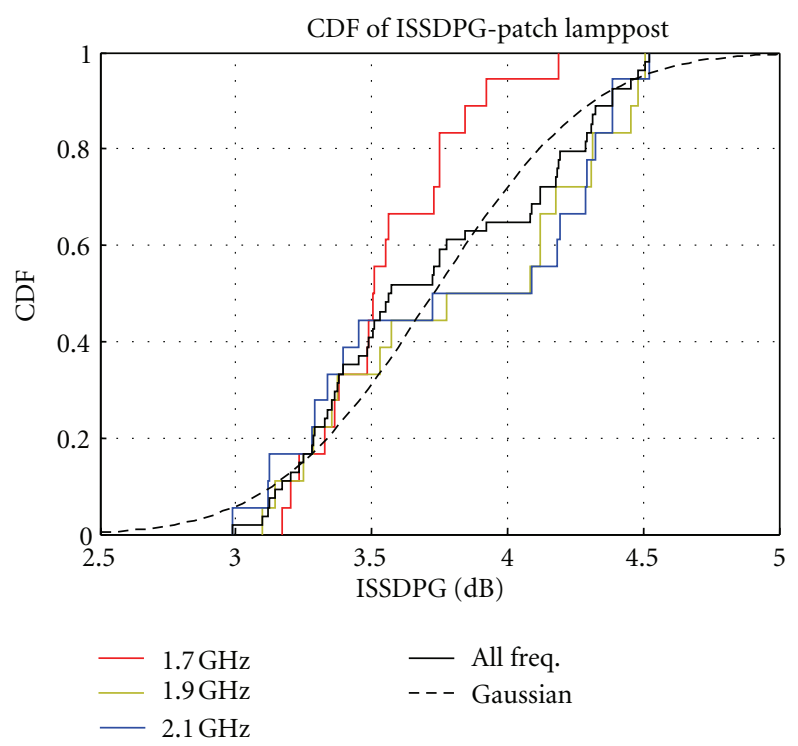

FIGURE 27: ISSDPG distribution for lamppost scenario.

relays, or base stations. The concept of the sector has been applied in order to focus the statistical analysis of the antenna pattern degradation by these disturbers in terms of wanted and unwanted radiation directions. Three parameters assessing the antenna performance and its degradation, which could be used in order to set up a statistical model, have been defined.

The results indicate that the lognormal distribution (Gaussian in $\mathrm{dB}$ ) is often approximately obeyed, as regards the power gains in the considered sector. This is true for the antenna-wall scenario, while in the antenna-shelter and antenna lamppost scenarios, the ISPG appears to follows the generalized Gaussian distribution. In most scenarios, except for the patch-lamp post scenario, the mean value of ISPG is less than that of a reference antenna, due to the reflection by surrounding disturbers. The ISSDPG follows well a lognormal distribution in the antenna-wall scenarios, within the frequency band from $1.7 \mathrm{GHz}$ to $2.0 \mathrm{GHz}$ for both directional and nondirectional antennas. For the other scenarios, its distribution shall be carefully investigated in future works. This statistical analysis of averages and deviations of the gain in the considered sector may be helpful in appreciating the problems posed by antennas deployment in small cells at street level, when the placement of antennas must be made in locations where strong disturbances are present. In the future, the frequency and angular correlation of radiation patterns will be investigated, and the goodness of fit for statistical parameter evaluation will be studied.

\section{Acknowledgments}

This work has been partly carried out with funding from the RECOSS project under the French competitiveness cluster "Systematic." The authors are grateful to Y. Lostanlen (SIRADEL) for his support and encouragement to the work. 


\section{References}

[1] Y. Jeong, T. Q. S. Quek, and H. Shin, "Beamforming optimization for multiuser two-tier networks," Journal of Communications and Networks, vol. 13, no. 4, pp. 327-338, 2011.

[2] V. Chandrasekhar, M. Kountouris, and J. G. Andrews, "Coverage in multi-antenna two-tier networks," IEEE Transactions on Wireless Communications, vol. 8, no. 10, pp. 5314-5327, 2009.

[3] V. Chandrasekhar and J. G. Andrews, "Uplink capacity and interference avoidance for two-tier femtocell networks," IEEE Transactions on Wireless Communications, vol. 8, no. 7, pp. 3498-3509, 2009.

[4] C. Roblin and M. A. Yousuf, "Statistical models of wideband and UWB omni-directional antennas based on a parametric modelling," in Proceedings of the 4th European Conference on Antennas and Propagation (EuCAP '10), Barcelona, Spain, April 2010.

[5] F. Molisch Andreas, F. Josef, and B. Ernst, "Pattern distortion of mobile radio base station antennas by antenna masts and roofs," in Proceedings of the 25th European Microwave Conference, vol. 1, pp. 71-76, September 1995.

[6] G. Stratis, C. A. Corral, S. Emami, S. Sibecas, G. Rasor, and R. Kipp, "Composite antenna pattern for realistic ray tracing simulations," in Proceedings of the IEEE International Antennas and Propagation Symposium and USNC/CNC/URSI North American Radio Science Meeting, pp. 106-109, USA, June 2003.

[7] R. L. Jesch, "Measured vehicular antenna performance," IEEE Transactions on Vehicular Technology, vol. 34, no. 2, pp. 97107, 1985.

[8] A. Sibille, J. Guterman, A. Moreira, and C. Peixeiro, "Performance evaluation of $2.4 \mathrm{GHz}$ laptop antennas using a joint antenna-channel statistical model," Technical Report TD-09930 28-30, Vienna, Austria, 2009.

[9] A. Sibille and C. Roblin, "Analysis and modelling of the randomness in terminals antennas," WAVES, pp. 39-48, 2010.

[10] M. A. Mellah, A. Sibille, C. Roblin, M. Nedil, and T. A. Denidni, "Statistical modeling of the "antenna-head" interaction," IEEE Antennas and Wireless Propagation Letters, vol. 10, pp. 454-457, 2011.

[11] CST: Microwave Studio, 2011th edition.

[12] http://www.wipl-d.com.

[13] I. Siomina, P. Värbrand, and D. Yaun, "Automated optimization of service coverage and base station antenna configuration in UMTS networks," IEEE Wireless Communications, vol. 13, no. 6, pp. 16-25, 2006.

[14] L. Song and J. Shen, Evolved Cellular Network Planning and Optimization for UMTS and LTE, CRC Press, London, UK, 1st edition, 2010.

[15] Dielectric Constants of Materials, Clipper Controls, 2007.

[16] B. H. Fleury, "First- and second-order characterization of direction dispersion and space selectivity in the radio channel," IEEE Transactions on Information Theory, vol. 46, no. 6, pp. 2027-2044, 2000. 

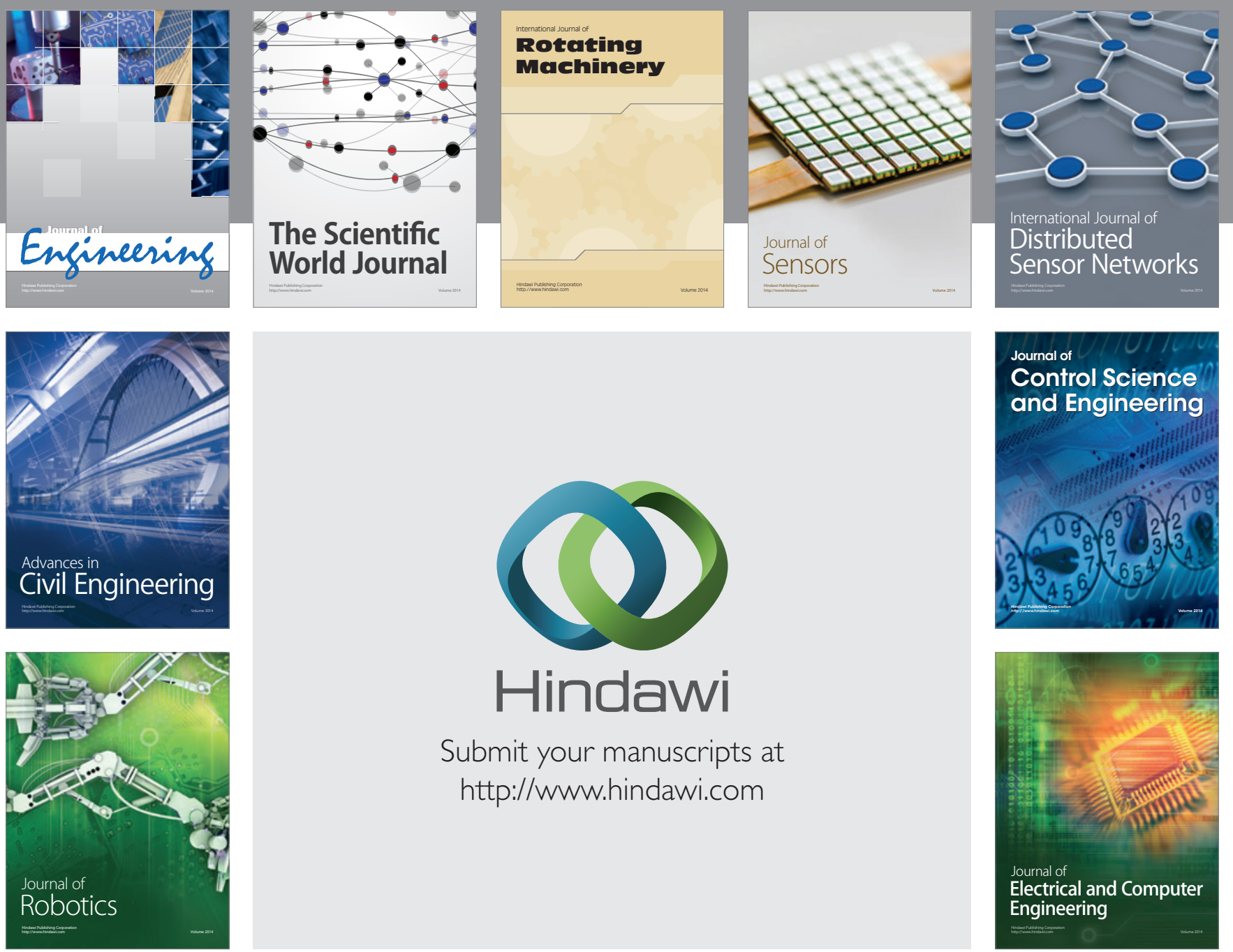

Submit your manuscripts at

http://www.hindawi.com
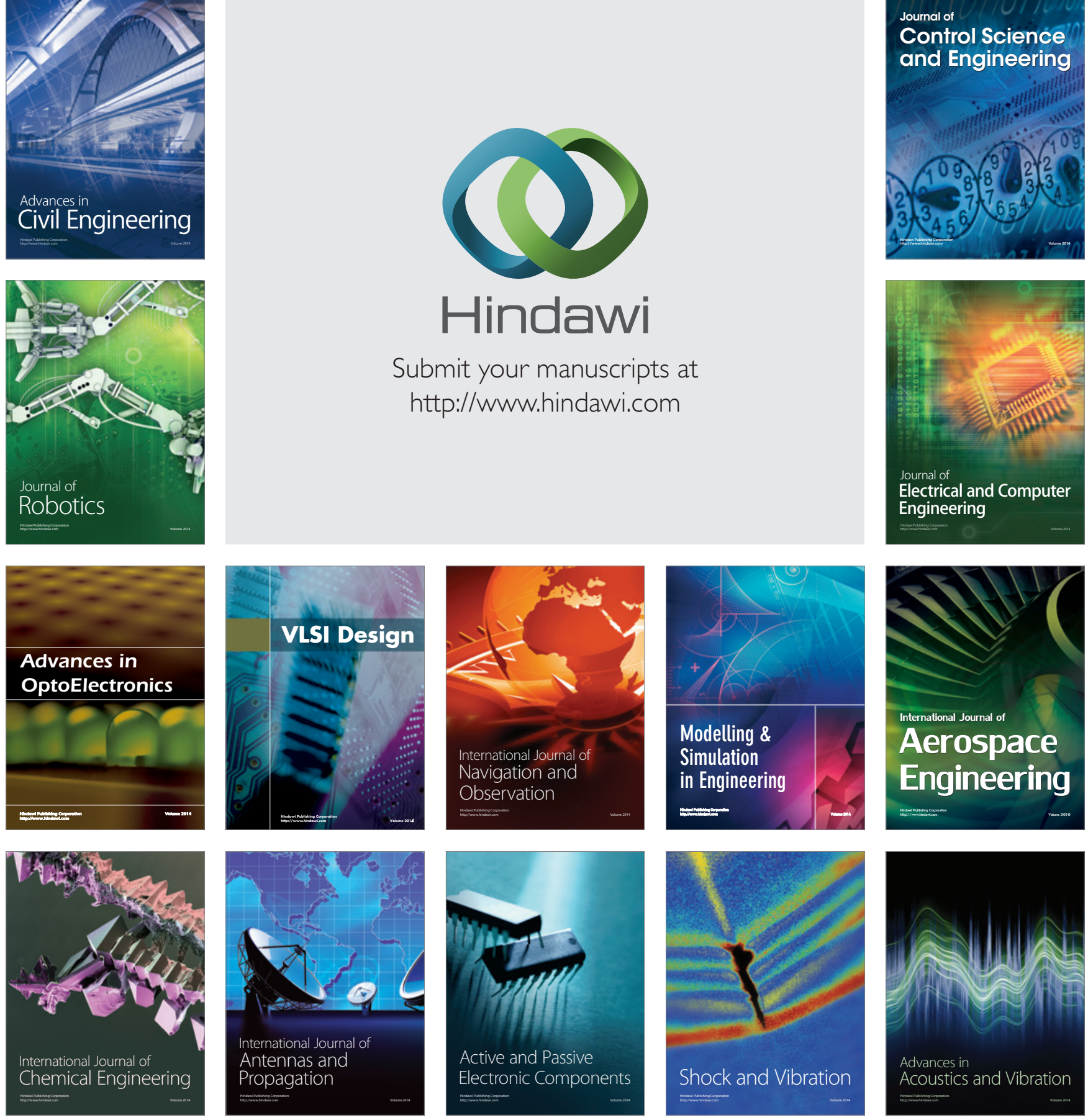